\title{
Physico-chemical Profile and Microbial Diversity During Bioconversion of Sugarcane Press Mud Using Bacterial Suspension
}

\author{
Tushar Chandra SARKER ${ }^{1}$, Mohammad Abdul MANNAN', Palash Chandra MONDOL², \\ Ahmad Humayan KABIR', Sarwar M. PARVEZ', Mohammad Firoz ALAM ${ }^{1 *}$ \\ ${ }^{1}$ University of Rajshahi, Department of Botany, Plant Biotechnology and Microbiology Laboratory, Rajshabi 6205, Bangladesh \\ ${ }^{2}$ Shanghai Jiao Tong University, School of Life Sciences and Biotechnology, Shanghai, China; falambt@ru.ac.bd (*corresponding author)
}

\begin{abstract}
This study was aimed at investigating the physico-chemical and microbial diversity for rapid composting of sugarcane press mud (PM) leading to organic manure. Five bacterial strains (Cellulomonas sp., Klebsiella sp., Proteus sp., Enterobacter sp., Salmonella sp.) were tested under in vivo conditions for bioconversion of PM using pile method. Results revealed that combined inoculation of bacterial consortia was found to be the best decomposer of PM resulting reduction of organic carbon content (26.75\%), C:N ratio (12.44\%). In parallel, it increased the nitrogen (2.34\%), phosphorous (1.15\%) and potassium (1.37\%) content along with the population of microorganisms i.e. bacteria, fungi and actinomycetes. However, the population of tested bacteria was gradually depleted after completion of PM decomposition together with pathogenic bacteria and fungi due to full conversion of carbon component into other minerals, i.e. N, P, K etc. Taken together, these findings certainly pinpoints the effective role of bacterial suspension for composting sugarcane press mud which the eventually be used as organic manure.
\end{abstract}

Keywords: bacterial consortia, bioconversion, composting, press mud, sugarcane

\section{Introduction}

For year's sugar industries faced a major challenge in proper utilization of the wastes generated in the process of sugar manufacturing in their plant. Therefore, there is considerable economic interest in the technology and development processes for effective utilization of these wastes (Zhang et al., 2000). As a result emphasis is now on aerobic composting, that converts wastes into organic manure rich in plant nutrients and humus and biodegradation of lignocellulosic wastes through an integrated system of composting with bio-inoculants (Singh and Sharma, 2002).

Physically, sugarcane is constituted by four major fractions, whose relative magnitude depends on the sugar agroindustrial process: fiber, nonsoluble solids, soluble solids, and water. The fiber is composed of the whole organic solid fraction, originally found in the cane's stem, and characterized by its marked heterogeneity (Triana et al., 1990). Sugarcane press mud is the residue of the filtration of sugarcane juice. Large amounts of press mud are released by the sugarcane industry and the disposal of this by-product is a major issue. Most of the part of these wastes is usually burnt in the field due to lack of proper management techniques, which creates severe environmental pollution and health hazards (Pandit et al., 2012).

Press mud contains fairly high quantities of plant nutrients that are essential for plants besides being a very ef- fective as soil amendment. Press mud contains appreciable of nitrogen, $\mathrm{Ca}, \mathrm{Mg}, \mathrm{S}$, trace element and organic matter. It also contains about $1 \%$ sugar, crude fiber and coagulated protein including cane wax, albuminoids, inorganic salts and particles (Mayalagu et al., 1983). Though press mud is very useful, now it is a serious problem because the lack of proper management. The improper management of this waste causes human and animal diseases, pollutes the air and soil environment and destroys natural ecosystem balance. Studies show that on an average, Bangladesh is producing about 0.85 million tons of sulphitation press mud every year from sulphitation process (Bokhtiar and Sakurai, 2005). Unfortunately, extensive research still needs to be undertaken for developing protocol for rapid degradation of press mud into enrichment compost.

Among the microbes, bacteria are the most important one for decomposing waste. Bacteria use press mud for their metabolism and finally they produce some simple and useful compounds from them which are important for soil health, plant growth and over all to keep well balance of natural ecosystem (Zaved et al., 2008). Moreover, efficiency of bacterial in bioconversion or organic compounds is well documented (Petre et al., 1999; Suhaimi et al., 2012). Unfortunately, knowledge on physico-chemical and microbial diversity of bacteria on bioconversion of sugarcane press mud is limited. Therefore, this study was conducted to monitor the chemical and biological changes during composting of sugarcane press mud with cattle manure 
in order to get high quality stabilized product within the goal of shortening the stabilization time.

\section{Materials and methods}

\section{Microbial source}

Five bacterial strains i.e. Cellulomonas sp. (HSMPM-2), Klebsiella sp. (HSMPM-4), Proteus sp. (HSMDP-2), Enterobacter sp. (HSMPM-3) and Salmonella sp. (HSMDS-3 were tested for in-vivo bioconversion/composting of press mud. These bacteria were identified in Plant Biotechnology and Microbiology Laboratory and are maintained as stock for experimentation.

\section{Collection of raw materials (press mud and cattle manure)}

Press mud (six month old) was collected in gunny bag from Harian Sugar Industry Ltd., Harian, Rajshahi, Bangladesh. Cattle manure (a mixture of liquid and solid forms) was also collected from the local private Cattle farm located at Kajla, Rajshahi City. Sub samples of cattle manure were air dried at $80^{\circ} \mathrm{C}$ for 24 hours, ground and passed through a $2.0 \mathrm{~mm}$ sieve. The samples were then stored in desiccators for further analysis and usages.

\section{Preparation and application of bacterial suspension}

Selected bacterial strains were separately cultured in LB (Luria Broth) media. A loop full of 4-day old colony of each bacterial strains was separately transferred to sterilize $200 \mathrm{ml} \mathrm{LB}$ broth in a $500 \mathrm{ml}$ conical flask kept on a orbital shaker $(110 \mathrm{rpm})$ at $28^{\circ} \mathrm{C}$ for 5 days. The culture flask was placed on shaker at $50 \mathrm{rpm}$ for 15 minutes at the end of incubation. The content of each flask was used as bacterial suspension $\left(2 \times 10^{6}\right)$ and was mixed properly with composting pile ratio of 100:500 grams of press mud. A control was used having no inoculation.

\section{Composting pile establishment}

Each pile (50 kg in weight) contained 2:1 ratio of press mud and cattle manure. The dimension of each pile was approximately $50 \mathrm{~cm}$ (width) $\times 50 \mathrm{~cm}$ (length) $\times 50 \mathrm{~cm}$ (height). The moisture content in each pile was adjusted to $60 \%$ before and during the composting process until the temperature in compost reached the ambient temperature. Once it was achieved, water supply was stopped though the composting process continued. Each compost pile was manually mixed with a shovel for about 10 minutes to turn the pile and provide aeration. This was done every 3-4 days until the compost piles reached maturity. The ambient temperature and the temperature within each pile at a depth of $15 \mathrm{~cm}$ from the surface of the piles were determined using a thermometer. The bacterial consortium was added as a layer wise. The composting piles were prepared as following combinations: $\mathrm{T}_{1}$ : Control (Without microorganism), $\mathrm{T}_{2}: \mathrm{PM}+$ Enterobacter sp. $+\mathrm{CM}, \mathrm{T}_{3}: \mathrm{PM}+$ Salmonella sp. $+\mathrm{CM}, \mathrm{T}_{4}: \mathrm{PM}+$ Proteus sp. $+\mathrm{CM}^{3}, \mathrm{~T}_{5}: \mathrm{PM}$
+ Klebsiella sp. $+\mathrm{CM}, \mathrm{T}_{6}: \mathrm{PM}+$ Cellulomonas sp. $+\mathrm{CM}$ and $\mathrm{T}_{7}: \mathrm{PM}+$ Consortium $+\mathrm{CM}$

Note: PM-Press mud, CM-Cattle manure; Consortium-Enterobacter sp. + Salmonella sp. + Proteus sp. + Klebsiella sp. + Cellulomonas sp.

\section{Analysis of physico-chemical parameters}

The compost samples were analyzed for the following parameters; temperature, moisture content, $\mathrm{pH}$ value, total organic matter, total organic carbon, total nitrogen and $\mathrm{C} / \mathrm{N}$ ratio throughout the composting period. Temperature of inoculated PM was observed by thermometer for every 7 days. (Tiquia and Tam, 1998). The moisture content was determined by oven dry method (Page et al., 1982). To determine the $\mathrm{pH}$ of the decomposed PM, $25 \mathrm{ml}$ of sterilized distilled water was added in $10 \mathrm{gm}$ decomposed PM kept in bottle at room temperature for 18 hours. The $\mathrm{pH}$ value was then measured using a digital $\mathrm{pH}$ meter having a glass electrode (Tiquia and Tam, 1998). Total organic carbon (expressed in \%) was determined by the Walkley and Black method (Walkley and Black, 1934). Furthermore, total nitrogen was analyzed followed by Kjeldal method (Black, 1965). Total phosphorus (TP) was determined spectrophotometrically (Fiske and Row Subha, 1925) while total potassium (TK) was detected by the flame emission technique (Pearson, 1952).

\section{Analysis of microbiological parameters}

Bacteria, fungi and actinomycetes samples were collected from each treatment separately and subsequently air dried for determining the population. Ten grams of air dried samples were serially diluted up to $10^{4}$ dilution and these diluted samples were then used for estimation. Bacteria $\left(10^{4}\right)$, fungi $\left(10^{2}\right)$ and actinomycetes $\left(10^{2}\right)$ cultured on nutrient agar, Martins Rose Bengal agar, kusters agar medium respectively, and were used for enumeration of these microorganisms. In addition, potato dextrose agar (PDA) medium used for enumeration of fungal pathogens. The petri plates were incubated at $30^{\circ} \mathrm{C}$ for a week, and population were counted and expressed per unit dry weight of substrate.

\section{Data analysis}

The experiment was carried in a randomized block design (RBD) having three replications. All statistical analysis was performed using SPSS (SPSS Inc.,Chicago, IL, USA) program. Results of all analysis were judged for its significant at $5 \%$ level.

\section{Results}

\section{Changes of moisture content (\%)}

At the initial stage of composting, the moisture content of all piles was around 62-64\%; however, it dropped gradually during composting time (Fig. 1). The moisture content of all piles decreased slowly to $38.82-46.62 \%$ at 


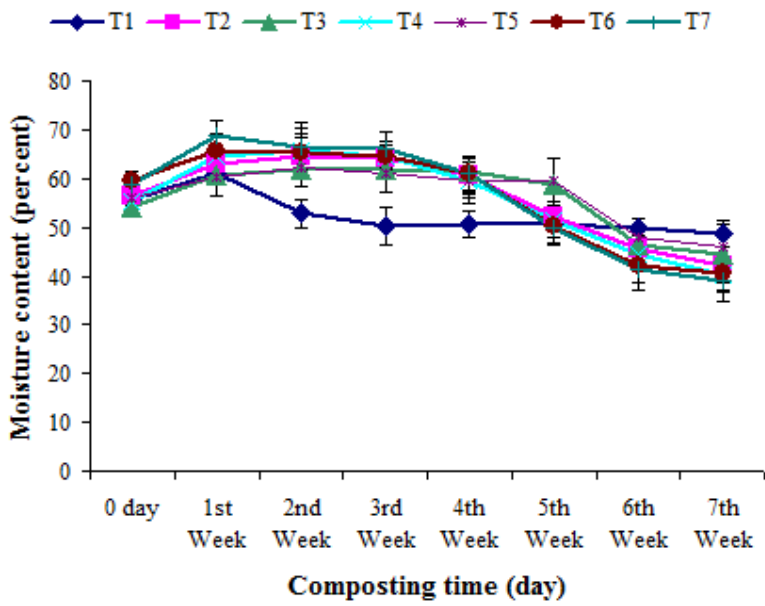

Fig. 1. Changes of Moisture content (\%) during composting of press mud. $\mathrm{T}_{1}$ : Control (Without microorganism); $\mathrm{T}_{2}: \mathrm{PM}+$ Enterobacter sp. $+\mathrm{CM} ; \mathrm{T}_{3}: \mathrm{PM}+$ Salmonella sp. $+\mathrm{CM} ; \mathrm{T}_{4}: \mathrm{PM}$ + Proteus sp. + CM; $\mathrm{T}_{5}: \mathrm{PM}+$ Klebsiella sp.+ CM; $\mathrm{T}_{6}: \mathrm{PM}+$ Cellulomonas sp. $+\mathrm{CM} ; \mathrm{T}_{7}: \mathrm{PM}+$ Consortium $+\mathrm{CM}$

the end of the composting period. It was considered that the piles achieved an acceptable level of mature compost, i.e., $\leq 50 \%$.

\section{Changes of $p H$}

The $\mathrm{pH}$ of all piles dropped from 7.8 to 5.5 during the first 14 days of composting (Fig. 2). After 21 days of composting, the $\mathrm{pH}$ of all piles increased and $\mathrm{pH}$ levels remained relatively unchanged after 49 days.

\section{Temperature profile}

The initial mean temperature of all piles was approximately $25^{\circ} \mathrm{C}$. Furthermore, high temperature $\left(64^{\circ} \mathrm{C}\right)$ continued to observe in all piles (except control) for about 14 days (Fig. 3) and then gradually dropped to $30.5^{\circ} \mathrm{C}$ at day 42. After day 42, the temperature varied within a narrow range until day 49 (Fig. 3).

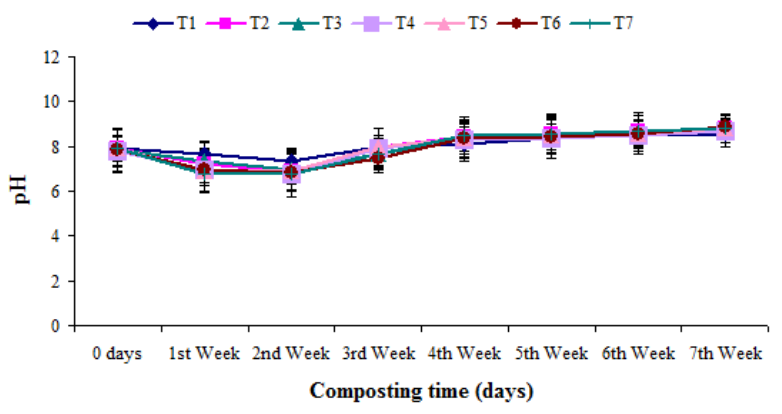

Fig. 2. Changes of $\mathrm{pH}$ during composting of press mud. $\mathrm{T}_{1}$ : Control (Without microorganism); $\mathrm{T}_{2}: \mathrm{PM}+$ Enterobacter $\mathrm{sp}$. + CM; $\mathrm{T}_{3}: \mathrm{PM}+$ Salmonella $\mathrm{sp} .+\mathrm{CM} ; \mathrm{T}_{4}: \mathrm{PM}+$ Proteus $\mathrm{sp} .+$ $\mathrm{CM} ; \mathrm{T}_{5}: \mathrm{PM}+$ Klebsiella sp. $+\mathrm{CM} ; \mathrm{T}_{6}: \mathrm{PM}+$ Cellulomonas $\mathrm{sp}$. $+\mathrm{CM} ; \mathrm{T}_{7}: \mathrm{PM}+$ Consortium+ CM.

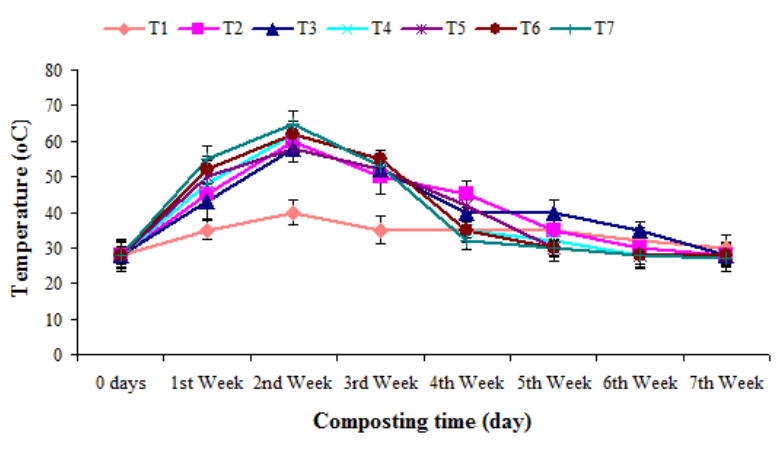

Fig. 3. Changes of Temperature during composting of press mud with cattle manure. $\mathrm{T}_{1}$ : Control (Without microorganism); $\mathrm{T}_{2}$ : $\mathrm{PM}+$ Enterobacter sp. $+\mathrm{CM} ; \mathrm{T}_{3}: \mathrm{PM}+$ Salmonella sp. $+\mathrm{CM}$; $\mathrm{T}_{4}: \mathrm{PM}+$ Proteus sp. $+\mathrm{CM} ; \mathrm{T}_{5}: \mathrm{PM}+$ Klebsiella sp. $+\mathrm{CM} ; \mathrm{T}_{6}:$ $\mathrm{PM}+$ Cellulomonas sp. $+\mathrm{CM} ; \mathrm{T}_{7}: \mathrm{PM}+$ Consortium $+\mathrm{CM}$

\section{Changes of organic carbon}

In general, percentage of organic carbon reduced significantly with increase in the period of decomposition in press mud up to end of 49 days. The initial organic carbon of press mud was $54-56 \%$. Among all the treatments, combinations of bacterial consortia $\left(T_{7}, \overline{\mathrm{x}}=26.75 \%\right)$ showed superior effect compared to control and other combinations. Irrespective of composting period, the organic carbon reduced significantly with increase in the period of decomposition up to end of 49 days. Maximum reduced organic carbon was recorded in bacterial consortia in composting period 49 days (ranged 36.27 to $18.86 \%$ ).

\section{Changes of nitrogen (\%)}

Inoculum levels were found to have significant effect on nitrogen content in composting of press mud. Unlike carbon content, nitrogen content was increased gradually (Tab. 2). Among all the treatments, bacterial consortia $\left(\mathrm{T}_{7}\right)$ showed maximum nitrogen content $(2.34 \%)$. Further, there was no significant difference in the nitrogen content observed among the composting period. It was also found that nitrogen content with increased period of decomposition was increased resulting highest value (1.97\%) at the end of 49 days compared to 7 days (1.08\%). Among the treatment combinations, maximum nitrogen content was observed in bacterial consortia (ranged 1.59 to $2.89 \%$ ) with incubation period of 49 days. This treatment was found superior (non-significant) than control (ranged 0.69 to $1.24 \%$ ) and other treatment combinations.

\section{Changes of carbon nitrogen ratio}

A significant reduction of $\mathrm{C}: \mathrm{N}$ ratio in the press mud compost was observed. Among all the treatments, bacterial consortia recorded significantly superior $(12.44 \%)$ over control (47.54\%) and other treatments (Tab. 3). Irrespective of incubation period, there was significant difference in C: $\mathrm{N}$ ratio in the compost. The C: $\mathrm{N}$ ratio reduced significantly with increase in the period of decomposition 
Tab. 1. Changes of organic carbon percent during composting of press mud with cattle manure

\begin{tabular}{ccccccccc}
\hline \multirow{2}{*}{ Treatments } & \multicolumn{7}{c}{ Number of Weeks } \\
\cline { 2 - 9 } & $1^{\text {st }}$ Week & $2^{\text {nd }}$ Week & $3^{\text {rd }}$ Week & $4^{\text {th }}$ Week & $5^{\text {th }}$ Week & $6^{\text {th }}$ Week & $7^{\text {th }}$ Week & Mean \\
\hline $\mathrm{T}_{1}:$ Control (Without microorganism) & $52.63 \mathrm{a}$ & $48.56 \mathrm{a}$ & $45.98 \mathrm{a}$ & $42.69 \mathrm{a}$ & $40.35 \mathrm{a}$ & $38.78 \mathrm{a}$ & $36.58 \mathrm{a}$ & $43.65 \mathrm{a}$ \\
$\mathrm{T}_{2}:$ PM+ Enterobacter sp.+ CM & $42.26 \mathrm{c}$ & $40.35 \mathrm{c}$ & $35.25 \mathrm{c}$ & $32.24 \mathrm{~d}$ & $30.28 \mathrm{~cd}$ & $27.36 \mathrm{c}$ & $25.96 \mathrm{c}$ & $33.38 \mathrm{~d}$ \\
$\mathrm{~T}_{3}: \mathrm{PM}+$ Salmonella sp.+ CM & $44.68 \mathrm{~b}$ & $41.12 \mathrm{c}$ & $38.69 \mathrm{~b}$ & $35.63 \mathrm{c}$ & $31.18 \mathrm{c}$ & $28.89 \mathrm{c}$ & $27.54 \mathrm{c}$ & $35.39 \mathrm{c}$ \\
$\mathrm{T}_{4}:$ PM + Proteus sp.+ CM & $40.63 \mathrm{c}$ & $38.52 \mathrm{~d}$ & $34.34 \mathrm{~d}$ & $31.19 \mathrm{~d}$ & $28.57 \mathrm{~d}$ & $25.57 \mathrm{~d}$ & $23.35 \mathrm{~d}$ & $31.74 \mathrm{~d}$ \\
$\mathrm{~T}_{5}:$ PM+ Klebsiella sp. $+\mathrm{CM}$ & $45.86 \mathrm{~b}$ & $42.23 \mathrm{~b}$ & $40.21 \mathrm{~b}$ & $38.67 \mathrm{~b}$ & $34.45 \mathrm{~b}$ & $31.24 \mathrm{~b}$ & $29.67 \mathrm{~b}$ & $37.48 \mathrm{~b}$ \\
$\mathrm{~T}_{6}: \mathrm{PM}+$ Cellulomonas sp. $+\mathrm{CM}$ & $38.53 \mathrm{~d}$ & $35.42 \mathrm{e}$ & $33.34 \mathrm{~d}$ & $28.86 \mathrm{e}$ & $25.21 \mathrm{e}$ & $23.34 \mathrm{e}$ & $20.19 \mathrm{e}$ & $29.27 \mathrm{e}$ \\
$\mathrm{T}_{7}:$ PM+ Consortium + CM & $36.27 \mathrm{e}$ & $33.22 \mathrm{f}$ & $30.28 \mathrm{e}$ & $26.24 \mathrm{f}$ & $22.16 \mathrm{f}$ & $20.19 \mathrm{f}$ & $18.86 \mathrm{e}$ & $26.75 \mathrm{f}$ \\
Mean & 42.98 & 39.92 & 36.87 & 33.65 & 30.32 & 27.91 & 26.02 & \\
\hline
\end{tabular}

Means in a column followed by the same letter $(s)$ are not significantly different according to DMRT $(p=0.05)$

Tab. 2. Changes of Nitrogen percent during composting of press mud with cattle manure

\begin{tabular}{|c|c|c|c|c|c|c|c|c|}
\hline \multirow{2}{*}{ Treatments } & \multicolumn{8}{|c|}{ Number of Weeks } \\
\hline & $1^{\text {st }}$ Week & $2^{\text {nd }}$ Week & $3^{\text {rd }}$ Week & $4^{\text {th }}$ Week & $5^{\text {th }}$ Week & $6^{\text {th }}$ Week & $7^{\text {th }}$ Week & Mean \\
\hline $\mathrm{T}_{1}$ : Control (Without microorganism) & $0.69 \mathrm{a}$ & $0.79 \mathrm{a}$ & $0.86 \mathrm{a}$ & $0.96 \mathrm{a}$ & $1.15 \mathrm{a}$ & $1.19 \mathrm{a}$ & $1.24 \mathrm{a}$ & $0.98 \mathrm{a}$ \\
\hline $\mathrm{T}_{2}: \mathrm{PM}+$ Enterobacter $\mathrm{sp} .+\mathrm{CM}$ & $0.98 \mathrm{a}$ & $1.12 \mathrm{a}$ & $1.26 \mathrm{a}$ & $1.48 \mathrm{a}$ & $1.68 \mathrm{a}$ & $1.78 \mathrm{a}$ & $1.87 \mathrm{a}$ & $1.45 \mathrm{a}$ \\
\hline $\mathrm{T}_{3}: \mathrm{PM}+$ Salmonella sp.+ CM & $0.94 \mathrm{a}$ & $0.98 \mathrm{a}$ & $1.12 \mathrm{a}$ & $1.24 \mathrm{a}$ & $1.45 \mathrm{a}$ & $1.54 \mathrm{a}$ & $1.64 \mathrm{a}$ & $1.27 \mathrm{a}$ \\
\hline $\mathrm{T}_{4}: \mathrm{PM}+$ Proteus sp. $+\mathrm{CM}$ & $1.12 \mathrm{a}$ & $1.34 \mathrm{a}$ & $1.59 \mathrm{a}$ & $1.68 \mathrm{a}$ & $1.87 \mathrm{a}$ & $1.95 \mathrm{a}$ & $2.14 \mathrm{a}$ & $1.67 \mathrm{a}$ \\
\hline $\mathrm{T}_{5}: \mathrm{PM}+$ Klebsiella sp. $+\mathrm{CM}$ & $0.89 \mathrm{a}$ & $0.96 \mathrm{a}$ & $1.05 \mathrm{a}$ & $1.16 \mathrm{a}$ & $1.34 \mathrm{a}$ & $1.46 \mathrm{a}$ & $1.56 \mathrm{a}$ & $1.20 \mathrm{a}$ \\
\hline $\mathrm{T}_{6}: \mathrm{PM}+$ Cellulomonas sp. $+\mathrm{CM}$ & $1.36 \mathrm{a}$ & $1.57 \mathrm{a}$ & $1.69 \mathrm{a}$ & $1.78 \mathrm{a}$ & $1.98 \mathrm{a}$ & $2.12 \mathrm{a}$ & $2.45 \mathrm{a}$ & $1.85 \mathrm{a}$ \\
\hline $\mathrm{T}_{7}: \mathrm{PM}+$ Consortium $+\mathrm{CM}$ & $1.59 \mathrm{a}$ & $1.96 \mathrm{a}$ & $2.18 \mathrm{a}$ & $2.39 \mathrm{a}$ & $2.49 \mathrm{a}$ & $2.86 \mathrm{a}$ & $2.89 \mathrm{a}$ & $2.34 \mathrm{a}$ \\
\hline Mean & 1.08 & 1.25 & 1.39 & 1.53 & 1.71 & 1.85 & 1.97 & \\
\hline
\end{tabular}

Means in a column followed by the same letter(s) are not significantly different according to DMRT $(p=0.05)$

Tab. 3. Changes of $\mathrm{C}: \mathrm{N}$ ratio during composting of press mud

\begin{tabular}{ccccccccc}
\hline \multirow{2}{*}{ Treatments } & \multicolumn{7}{c}{ Number of Weeks } \\
\cline { 2 - 9 } & $1^{\text {st }}$ Week & $2^{\text {nd }}$ Week & $3^{\text {rd }}$ Week & $4^{\text {th }}$ Week & $5^{\text {th }}$ Week & $6^{\text {th }}$ Week & $7^{\text {th }}$ Week & Mean \\
\hline $\mathrm{T}_{1}:$ Control (Without microorganism) & $76.27 \mathrm{a}$ & $61.46 \mathrm{a}$ & $53.46 \mathrm{a}$ & $44.46 \mathrm{a}$ & $35.09 \mathrm{a}$ & $32.59 \mathrm{a}$ & $29.5 \mathrm{a}$ & $47.54 \mathrm{a}$ \\
$\mathrm{T}_{2}: \mathrm{PM}+$ Enterobacter sp. $+\mathrm{CM}$ & $43.12 \mathrm{~d}$ & $36.02 \mathrm{~d}$ & $27.98 \mathrm{~d}$ & $21.78 \mathrm{~d}$ & $18.03 \mathrm{~d}$ & $15.37 \mathrm{~d}$ & $13.88 \mathrm{~d}$ & $25.17 \mathrm{~d}$ \\
$\mathrm{~T}_{3}: \mathrm{PM}+$ Salmonella sp.+ CM & $47.53 \mathrm{c}$ & $41.96 \mathrm{c}$ & $34.54 \mathrm{c}$ & $28.74 \mathrm{c}$ & $21.50 \mathrm{c}$ & $18.76 \mathrm{c}$ & $16.79 \mathrm{c}$ & $29.97 \mathrm{c}$ \\
$\mathrm{T}_{4}: \mathrm{PM}+$ Proteus sp. $+\mathrm{CM}$ & $36.28 \mathrm{e}$ & $28.75 \mathrm{e}$ & $21.59 \mathrm{e}$ & $18.57 \mathrm{e}$ & $15.28 \mathrm{e}$ & $13.11 \mathrm{e}$ & $10.91 \mathrm{e}$ & $20.64 \mathrm{e}$ \\
$\mathrm{T}_{5}: \mathrm{PM}+$ Klebsiella sp. $+\mathrm{CM}$ & $51.53 \mathrm{~b}$ & $43.98 \mathrm{~b}$ & $38.29 \mathrm{~b}$ & $33.34 \mathrm{~b}$ & $25.70 \mathrm{~b}$ & $21.39 \mathrm{~b}$ & $19.02 \mathrm{~b}$ & $33.32 \mathrm{~b}$ \\
$\mathrm{~T}_{6}: \mathrm{PM}+$ Cellulomonas sp. $+\mathrm{CM}$ & $28.33 \mathrm{f}$ & $22.56 \mathrm{f}$ & $19.73 \mathrm{f}$ & $16.21 \mathrm{f}$ & $12.73 \mathrm{f}$ & $11.01 \mathrm{f}$ & $8.24 \mathrm{f}$ & $16.97 \mathrm{f}$ \\
$\mathrm{T}_{7}: \mathrm{PM}+$ Consortium $+\mathrm{CM}$ & $22.81 \mathrm{~g}$ & $16.95 \mathrm{~g}$ & $13.88 \mathrm{~g}$ & $10.97 \mathrm{~g}$ & $8.89 \mathrm{~g}$ & $7.06 \mathrm{~g}$ & $6.53 \mathrm{f}$ & $12.44 \mathrm{~g}$ \\
Mean & 43.71 & 35.95 & 29.93 & 24.87 & 19.61 & 17.01 & 14.98 \\
\hline
\end{tabular}

Means in a column followed by the same letter(s) are not significantly different according to DMRT $(p=0.05)$

and it was lowest at the end of 49days (14.98\%) than at 7 days (43.71\%). Maximum reduced C: $\mathrm{N}$ ratio was recorded in bacterial consortia of 49 days (ranged 22.81 to $6.53 \%$ ), which was significantly superior over control (ranged 76.27 to $29.5 \%$ ) and all other treatment combinations (Tab. 3).

\section{Changes of phosphorus (\%)}

Irrespective of inoculums levels, there was significant difference on the phosphorus content of press mud compost. Among all the treatment combinations, bacterial consortia $\left(\mathrm{T}_{7}\right)$ were found $(1.15 \%)$ superior (non-significant) over control (0.39\%) and other treatments (Tab. 4). Among incubation period, there was significantly increase in phosphorous content with increase in the period of incubation. Maximum phosphorous content was recorded at 49 days (1.46\%) than at 7 days of incubation period $(0.58 \%)$. Maximum phosphorous content was recorded in bacterial consortia $\left(T_{7}\right)$ with incubation period of 49 days (1.09\%).

\section{Changes of potassium (\%)}

Inoculation levels were found to have significant effect on potassium content, among all the treatments (Tab. 5). Bacterial consortia $\left(\mathrm{T}_{7}\right)$ inoculation $(1.37 \%)$ was not significantly superior over control $\left(\mathrm{T}_{1}, 0.58 \%\right)$ and other treatment. Irrespective of composting period, the significant difference was found in potassium content in PM compost. The potassium content increased significantly with increase in the period of decomposition and it was highest at the end of 49 days. Among all the inoculums combinations, maximum potassium content was observed 
Tab. 4. Changes of Phosphorus percent during composting of press mud with cattle manure

\begin{tabular}{ccccccccc}
\hline \multirow{2}{*}{ Treatments } & \multicolumn{7}{c}{ Number of Weeks } \\
\cline { 2 - 9 } & $1^{\text {st }}$ Week & $2^{\text {nd }}$ Week & $3^{\text {rd }}$ Week & $4^{\text {th }}$ Week & $5^{\text {th }}$ Week & $6^{\text {th }}$ Week & $7^{\text {th }}$ Week & Mean \\
\hline $\mathrm{T}_{1}:$ Control (Without microorganism) & $0.26 \mathrm{~g}$ & $0.29 \mathrm{~g}$ & $0.31 \mathrm{~g}$ & $0.39 \mathrm{a}$ & $0.41 \mathrm{a}$ & $0.48 \mathrm{a}$ & $0.58 \mathrm{a}$ & $0.39 \mathrm{a}$ \\
$\mathrm{T}_{2}:$ PM+ Enterobacter sp.+ CM & $0.45 \mathrm{~d}$ & $0.56 \mathrm{~d}$ & $0.68 \mathrm{~d}$ & $0.79 \mathrm{a}$ & $0.84 \mathrm{a}$ & $0.96 \mathrm{a}$ & $1.12 \mathrm{a}$ & $0.77 \mathrm{a}$ \\
$\mathrm{T}_{3}: \mathrm{PM}+$ Salmonella sp.+ CM & $0.39 \mathrm{e}$ & $0.49 \mathrm{e}$ & $0.54 \mathrm{e}$ & $0.68 \mathrm{a}$ & $0.75 \mathrm{a}$ & $0.81 \mathrm{a}$ & $0.98 \mathrm{a}$ & $0.66 \mathrm{a}$ \\
$\mathrm{T}_{4}: \mathrm{PM}+$ Proteus sp.+ CM & $0.59 \mathrm{c}$ & $0.64 \mathrm{c}$ & $0.79 \mathrm{c}$ & $0.87 \mathrm{a}$ & $0.91 \mathrm{a}$ & $0.99 \mathrm{a}$ & $1.21 \mathrm{a}$ & $0.86 \mathrm{a}$ \\
$\mathrm{T}_{5}: \mathrm{PM}+$ Klebsiella sp. $+\mathrm{CM}$ & $0.29 \mathrm{f}$ & $0.39 \mathrm{f}$ & $0.48 \mathrm{f}$ & $0.59 \mathrm{a}$ & $0.67 \mathrm{a}$ & $0.78 \mathrm{a}$ & $0.95 \mathrm{a}$ & $0.59 \mathrm{a}$ \\
$\mathrm{T}_{6}: \mathrm{PM}+$ Cellulomonas sp. $+\mathrm{CM}$ & $0.64 \mathrm{~b}$ & $0.75 \mathrm{~b}$ & $0.87 \mathrm{~b}$ & $0.98 \mathrm{a}$ & $1.24 \mathrm{a}$ & $1.26 \mathrm{a}$ & $1.32 \mathrm{a}$ & $1.01 \mathrm{a}$ \\
$\mathrm{T}_{7}: \mathrm{PM}+$ Consortium $+\mathrm{CM}$ & $0.78 \mathrm{a}$ & $0.89 \mathrm{a}$ & $0.98 \mathrm{a}$ & $1.12 \mathrm{a}$ & $1.38 \mathrm{a}$ & $1.41 \mathrm{a}$ & $1.46 \mathrm{a}$ & $1.15 \mathrm{a}$ \\
Mean & 0.49 & 0.57 & 0.66 & 0.77 & 0.89 & 0.96 & 1.09 & \\
\hline
\end{tabular}

Means in a column followed by the same letter $(s)$ are not significantly different according to DMRT ( $p=0.05)$

Tab. 5. Changes of Potassium percent during composting of press mud with cattle manure

\begin{tabular}{|c|c|c|c|c|c|c|c|c|}
\hline \multirow{2}{*}{ Treatments } & \multicolumn{8}{|c|}{ Number of Weeks } \\
\hline & $1^{\text {st }}$ Week & $2^{\text {nd }}$ Week & $3^{\text {rd }}$ Week & $4^{\text {th }}$ Week & $5^{\text {th }}$ Week & $6^{\text {th }}$ Week & $7^{\text {th }}$ Week & Mean \\
\hline $\mathrm{T}_{1}$ :Control (Without microorganism) & $0.36 \mathrm{e}$ & $0.42 \mathrm{~g}$ & $0.49 \mathrm{a}$ & $0.54 \mathrm{a}$ & $0.68 \mathrm{a}$ & $0.76 \mathrm{a}$ & $0.79 \mathrm{a}$ & $0.58 \mathrm{a}$ \\
\hline $\mathrm{T}_{2}: \mathrm{PM}+$ Enterobacter $\mathrm{sp} .+\mathrm{CM}$ & $0.42 \mathrm{c}$ & $0.65 \mathrm{~d}$ & $0.75 \mathrm{a}$ & $0.86 \mathrm{a}$ & $0.98 \mathrm{a}$ & $1.16 \mathrm{a}$ & $1.26 \mathrm{a}$ & $0.87 \mathrm{a}$ \\
\hline $\mathrm{T}_{3}: \mathrm{PM}+$ Salmonella sp.+ CM & $0.39 \mathrm{~d}$ & $0.53 \mathrm{e}$ & $0.69 \mathrm{a}$ & $0.79 \mathrm{a}$ & $0.89 a$ & $0.98 \mathrm{a}$ & $1.12 \mathrm{a}$ & $0.77 \mathrm{a}$ \\
\hline $\mathrm{T}_{4}: \mathrm{PM}+$ Proteus sp.+ CM & $0.52 \mathrm{a}$ & $0.74 \mathrm{c}$ & $0.85 \mathrm{a}$ & $0.98 \mathrm{a}$ & $1.16 \mathrm{a}$ & $1.24 \mathrm{a}$ & $1.45 \mathrm{a}$ & $0.99 \mathrm{a}$ \\
\hline $\mathrm{T}_{5}:$ PM+ Klebsiella sp. $+\mathrm{CM}$ & $0.36 \mathrm{e}$ & $0.49 \mathrm{f}$ & $0.59 \mathrm{a}$ & $0.69 \mathrm{a}$ & $0.79 \mathrm{a}$ & $0.86 \mathrm{a}$ & $0.96 \mathrm{a}$ & $0.68 \mathrm{a}$ \\
\hline $\mathrm{T}_{6}: \mathrm{PM}+$ Cellulomonas sp. $+\mathrm{CM}$ & $0.46 b$ & $0.89 \mathrm{~b}$ & $0.98 \mathrm{a}$ & $1.26 \mathrm{a}$ & $1.48 \mathrm{a}$ & $1.68 \mathrm{a}$ & $1.72 \mathrm{a}$ & $1.21 \mathrm{a}$ \\
\hline $\mathrm{T}_{7}: \mathrm{PM}+$ Consortium $+\mathrm{CM}$ & $0.51 \mathrm{a}$ & $0.96 \mathrm{a}$ & $1.12 \mathrm{a}$ & $1.37 \mathrm{a}$ & $1.67 \mathrm{a}$ & $1.96 \mathrm{a}$ & $1.98 \mathrm{a}$ & $1.37 \mathrm{a}$ \\
\hline Mean & 0.43 & 0.67 & 0.78 & 0.92 & 1.09 & 1.24 & 1.33 & \\
\hline
\end{tabular}

Means in a column followed by the same letter(s) are not significantly different according to DMRT $(p=0.05)$

in bacterial consortia inoculation $(1.33 \%)$ with compost- Discussion

ing period of 49 days.

Population studies of bacteria, fungi and actinomyacetes during composting of press mud

The highest microbial load was observed in the composting of press mud inoculated with a bacterial consortium $\left(\mathrm{T}_{7}\right)$. The total bacterial population increased $\left(90.57 \times 10^{4} / \mathrm{gm}\right)$ at 49 days. Similarly, the fungal population was also increased $\left(72.72 \times 10^{2} / \mathrm{gm}\right)$ at 49 days. However, the actinomycetes population was maximum $\left(48.57 \times 10^{2} / \mathrm{gm}\right)$ at 49 days (Tab. 6-8). Similarly, the compost sample was tested for the presence of pathogenic microorganisms by following the serial dilution technique and plating technique. However it was observed that there were no pathogenic bacteria and fungi in the samples.

\section{Physico-chemical parameters}

For bioconversion of sugarcane press mud the moisture content (\%) of all piles was around 62-64\% and dropped gradually during composting time. The moisture content of all piles decreased slowly to around $38.82-46.62 \%$ at the end of the composting period. A decrease in moisture content was due to the release of moisture from the compost pile through water evaporation as a result of the heat generated from microbial activities during composting. In a similar study, Moqsud (2010) showed that the maximum moisture content reached nearly $67 \%$ before modification and about $60 \%$ after the modification of the barrel. When the moisture contents drop much below $40 \%$, the composting process becomes slow (Goyal et al., 2005). At the

Tab. 6. Changes of bacterial population $\left(\mathrm{CFU} \times 10^{4} / \mathrm{g}\right)$ during composting of Press mud with cattle manure

\begin{tabular}{|c|c|c|c|c|c|c|c|c|}
\hline \multirow{2}{*}{ Treatments } & \multicolumn{8}{|c|}{ Number of Weeks } \\
\hline & $1^{\text {st }}$ Week & $2^{\text {nd }}$ Week & $3^{\text {rd }}$ Week & $4^{\text {th }}$ Week & $5^{\text {th }}$ Week & $6^{\text {th }}$ Week & $7^{\text {th }}$ Week & Mean \\
\hline $\mathrm{T}_{1}$ : Control (Without microorganism) & $18 \mathrm{f}$ & $28 \mathrm{f}$ & $48 \mathrm{~g}$ & $68 \mathrm{f}$ & $59 \mathrm{~g}$ & $52 \mathrm{~g}$ & $48 \mathrm{f}$ & $45.85 \mathrm{~g}$ \\
\hline $\mathrm{T}_{2}: \mathrm{PM}+$ Enterobacter sp.+ CM & $38 \mathrm{~d}$ & $48 \mathrm{~d}$ & $68 \mathrm{~d}$ & $89 \mathrm{~d}$ & $78 \mathrm{~d}$ & $74 d$ & $68 c$ & $66.14 \mathrm{~d}$ \\
\hline $\mathrm{T}_{3}: \mathrm{PM}+$ Salmonella sp.+ CM & $35 \mathrm{e}$ & $45 \mathrm{e}$ & $66 \mathrm{e}$ & $87 \mathrm{e}$ & $76 \mathrm{e}$ & $69 \mathrm{e}$ & $62 \mathrm{~d}$ & $62.86 \mathrm{e}$ \\
\hline $\mathrm{T}_{4}: \mathrm{PM}+$ Proteus sp.+ CM & $45 c$ & $65 c$ & $85 c$ & $108 \mathrm{c}$ & $98 \mathrm{c}$ & $85 c$ & $78 \mathrm{~b}$ & $80.57 c$ \\
\hline $\mathrm{T}_{5}: \mathrm{PM}+$ Klebsiella sp. $+\mathrm{CM}$ & $34 \mathrm{e}$ & $44 \mathrm{e}$ & $64 \mathrm{f}$ & $86 \mathrm{e}$ & $70 \mathrm{f}$ & $64 \mathrm{f}$ & $59 \mathrm{e}$ & $60.14 \mathrm{f}$ \\
\hline $\mathrm{T}_{6}: \mathrm{PM}+$ Cellulomonas sp. $+\mathrm{CM}$ & $48 \mathrm{~b}$ & $68 \mathrm{~b}$ & $88 \mathrm{~b}$ & $112 \mathrm{~b}$ & $105 \mathrm{~b}$ & $98 \mathrm{~b}$ & $78 \mathrm{~b}$ & $85.29 \mathrm{~b}$ \\
\hline $\mathrm{T}_{7}: \mathrm{PM}+$ Consortium $+\mathrm{CM}$ & $51 \mathrm{a}$ & $73 \mathrm{a}$ & $92 \mathrm{a}$ & $119 a$ & $109 a$ & $105 \mathrm{a}$ & $85 a$ & $90.57 \mathrm{a}$ \\
\hline Mean & 38.43 & 53 & 73 & 95.57 & 85 & 78.14 & 68.29 & \\
\hline
\end{tabular}

Means in a column followed by the same letter(s) are not significantly different according to DMRT $(p=0.05)$ 
Tab. 7. Changes of fungal population $\left(\mathrm{CFU} \times 10^{2} / \mathrm{g}\right)$ during composting of press mud with cattle manure

\begin{tabular}{|c|c|c|c|c|c|c|c|c|}
\hline \multirow{2}{*}{ Treatments } & \multicolumn{8}{|c|}{ Number of Weeks } \\
\hline & $1^{\text {st }}$ Week & $2^{\text {nd }}$ Week & $3^{\text {rd }}$ Week & $4^{\text {th }}$ Week & $5^{\text {th }}$ Week & $6^{\text {th }}$ Week & $7^{\text {th }}$ Week & Mean \\
\hline $\mathrm{T}_{1}$ : Control (Without microorganism) & $28 \mathrm{f}$ & $36 \mathrm{e}$ & $46 \mathrm{e}$ & $57 \mathrm{f}$ & $52 \mathrm{~g}$ & $49 \mathrm{~g}$ & $45 \mathrm{~g}$ & $44.71 \mathrm{f}$ \\
\hline $\mathrm{T}_{2}: \mathrm{PM}+$ Enterobacter $\mathrm{sp} .+\mathrm{CM}$ & $41 \mathrm{c}$ & $51 \mathrm{c}$ & $62 \mathrm{~d}$ & $73 d$ & $69 \mathrm{~d}$ & $65 d$ & $61 \mathrm{~d}$ & $60.29 d$ \\
\hline $\mathrm{T}_{3}: \mathrm{PM}+$ Salmonella $\mathrm{sp} .+\mathrm{CM}$ & $39 \mathrm{~d}$ & $49 d$ & $63 \mathrm{~d}$ & $68 \mathrm{e}$ & $61 \mathrm{f}$ & $60 \mathrm{e}$ & $55 \mathrm{e}$ & $56.43 \mathrm{e}$ \\
\hline $\mathrm{T}_{4}: \mathrm{PM}+$ Proteus sp.+ CM & $49 a$ & $59 \mathrm{~b}$ & $71 \mathrm{c}$ & $83 \mathrm{~b}$ & $75 \mathrm{c}$ & $69 c$ & $63 \mathrm{c}$ & $67 c$ \\
\hline $\mathrm{T}_{5}: \mathrm{PM}+$ Klebsiella sp. $+\mathrm{CM}$ & $36 \mathrm{e}$ & $48 \mathrm{~d}$ & $62 \mathrm{~d}$ & $74 d$ & $64 \mathrm{e}$ & $55 \mathrm{f}$ & $53 \mathrm{f}$ & $56 \mathrm{e}$ \\
\hline $\mathrm{T}_{6}: \mathrm{PM}+$ Cellulomonas sp. $+\mathrm{CM}$ & $46 \mathrm{~b}$ & $59 \mathrm{~b}$ & $73 b$ & $81 \mathrm{c}$ & $78 \mathrm{~b}$ & $75 b$ & $71 \mathrm{~b}$ & $69 \mathrm{~b}$ \\
\hline $\mathrm{T}_{7}: \mathrm{PM}+$ Consortium $+\mathrm{CM}$ & $49 \mathrm{a}$ & $62 \mathrm{a}$ & $75 a$ & $86 a$ & $82 \mathrm{a}$ & $79 a$ & $76 a$ & $72.72 \mathrm{a}$ \\
\hline Mean & 41.14 & 52 & 64.58 & 74.58 & 68.71 & 64.58 & 60.57 & \\
\hline
\end{tabular}

Means in a column followed by the same letter $(s)$ are not significantly different according to DMRT $(p=0.05)$

Tab. 8. Changes of Actinomycetes population $\left(\mathrm{CFU} \times 10^{2} / \mathrm{g}\right)$ during composting of press mud with cattle manure

\begin{tabular}{cccccccccc}
\hline \multirow{2}{*}{ Treatments } & \multicolumn{7}{c}{ Number of Weeks } \\
\cline { 2 - 10 } & $1^{\text {st }}$ Week & $2^{\text {nd }}$ Week & $3^{\text {rd }}$ Week & $4^{\text {th }}$ Week & $5^{\text {th }}$ Week & $6^{\text {th }}$ Week & $7^{\text {th }}$ Week & Mean \\
\hline $\mathrm{T}_{1}:$ Control (Without microorganism) & $16 \mathrm{~g}$ & $25 \mathrm{f}$ & $30 \mathrm{~g}$ & $35 \mathrm{~g}$ & $31 \mathrm{e}$ & $29 \mathrm{~g}$ & $25 \mathrm{~g}$ & $27.29 \mathrm{f}$ \\
$\mathrm{T}_{2}:$ PM+ Enterobacter sp.+ CM & $20 \mathrm{e}$ & $32 \mathrm{~d}$ & $41 \mathrm{~d}$ & $48 \mathrm{~d}$ & $45 \mathrm{c}$ & $43 \mathrm{~d}$ & $40 \mathrm{~d}$ & $38.43 \mathrm{~d}$ \\
$\mathrm{~T}_{3}: \mathrm{PM}+$ Salmonella sp.+ CM & $18 \mathrm{f}$ & $28 \mathrm{e}$ & $39 \mathrm{e}$ & $43 \mathrm{e}$ & $40 \mathrm{~d}$ & $38 \mathrm{e}$ & $35 \mathrm{e}$ & $34.43 \mathrm{e}$ \\
$\mathrm{T}_{4}: \mathrm{PM}+$ Proteus sp.+ CM & $22 \mathrm{~d}$ & $36 \mathrm{c}$ & $45 \mathrm{c}$ & $52 \mathrm{c}$ & $50 \mathrm{~b}$ & $46 \mathrm{c}$ & $43 \mathrm{c}$ & $42 \mathrm{c}$ \\
$\mathrm{T}_{5}: \mathrm{PM}+$ Klebsiella sp. $+\mathrm{CM}$ & $26 \mathrm{c}$ & $29 \mathrm{e}$ & $35 \mathrm{f}$ & $41 \mathrm{f}$ & $39 \mathrm{~d}$ & $36 \mathrm{f}$ & $33 \mathrm{f}$ & $34.14 \mathrm{e}$ \\
$\mathrm{T}_{6}: \mathrm{PM}+$ Cellulomonas sp. $+\mathrm{CM}$ & $28 \mathrm{~b}$ & $38 \mathrm{~b}$ & $49 \mathrm{~b}$ & $54 \mathrm{~b}$ & $51 \mathrm{~b}$ & $48 \mathrm{~b}$ & $45 \mathrm{~b}$ & $44.73 \mathrm{~b}$ \\
$\mathrm{~T}_{7}: \mathrm{PM}+$ Consortium $+\mathrm{CM}$ & $31 \mathrm{a}$ & $45 \mathrm{a}$ & $51 \mathrm{a}$ & $58 \mathrm{a}$ & $55 \mathrm{a}$ & $52 \mathrm{a}$ & $48 \mathrm{a}$ & $48.57 \mathrm{a}$ \\
Mean & 23 & 33.29 & 41.43 & 47.29 & 44.43 & 41.71 & 38.43 & \\
\hline
\end{tabular}

Means in a column followed by the same letter(s) are not significantly different according to DMRT $(p=0.05)$

initial stage ( 5 -12 days), the bacterial activities and the decomposition of organic waste were more significant. The values of moisture content were also high in that period and it decreased after some days (15-25 days) as the bacterial activities decreased at that time.

Temperature is one of the key indicators of composting. When microbes degrade the waste, heat is produced and temperature increase with decomposition process and rising temperature accelerate plant residue degradation (Moqsud, 2003). Tchobanoglous (1977) also reported increase of temperature form initial day of composting up to two or three week but remained constant after that.

The $\mathrm{pH}$ of all piles dropped from approximately 7.8 to 5.5 during the first 14 days of composting. A large $\mathrm{pH}$ drop in the compost piles at the initial stage of composting might be due to the fact that organic carbon degraded to organic acid by the acid formation bacteria existing in the compost pile. After 21 days of composting, the $\mathrm{pH}$ of all piles increased and $\mathrm{pH}$ levels remained relatively unchanged after day 49 . Similar pattern of $\mathrm{pH}$ change was recorded by Kalamdhad and Kazmi (2009). The increase of $\mathrm{pH}$ in composting piles during the composting process could be due to the production of ammonium as a result of the amonification process.

In this study, the initial organic carbon content was 54-56\%, which was decreased to $26.75 \%$ with the decomposition of press mud due to the inoculated microbial inoculants bringing down substantial decrease in organic carbon. Among all the treatment combinations of bacte- rial consortia $\left(T_{7}, 26.75 \%\right)$, which showed significantly superior over control $\left(\mathrm{T}_{1}, 43.65 \%\right)$ and over all other treatment combinations (Tab. 1). The results clearly indicated that, all the inoculation of treatments was found to have significant effect on organic carbon. These results are in accordance with the observations of Gupta et al. (2004). Imam and Sharanappa (2002), who also reported decrease in the organic carbon when poultry manure was composted with different crop residues. The changes in organic carbon during the decomposition process indicated that the rate of loss of organic carbon in press mud was higher up to 49 th days compare to the control. This higher rate of decomposition in press mud was found to be due to the higher nitrogen content.

In this study the nitrogen content increased with decomposition. At the end of 49th day of composting, the $\mathrm{N}$ was found to be $2.34 \%$ in press mud compost. The increase in nitrogen content during composting might be a direct manifestation of mass loss and carbon loss (Nagarajan, 1985; Rasal et al., 1990; Singh et al., 1992; Maleena, 1998).

Initially, in press mud material $\mathrm{C}: \mathrm{N}$ ratio was higher, which with further decomposition decreased to minimum $12.44 \mathrm{~g}$. This decrease in C: $\mathrm{N}$ ratio was due to higher $\mathrm{N}$ content and faster rate of decomposition due to the activity of introduced bacterial strains. The reason might be the presence of bacteria that produce more cellulase than fungi. Several workers have suggested that inoculation of bacterial cultures resulted in rapid decomposition with 
352

decreases in C:N ratio (Murkate et al., 1992; Singh et al., 1992; Jagadeesh et al., 1996).

The phosphorous and potassium content in press mud compost showed changes which we assume due to the decomposition. At the end of 49th day, the phosphorous and potassium content was higher $1.15 \%$ and $1.37 \%$ in press mud compost. The nutrient content of compost showed that the better nutrient levels of concentration compared to control. This is probably because of quick microbial activity leading to decrease in volume of the material. Similar results found by Nagarajan (1985), Anandavalli et al. (1998) and Imam and Sharanappa (2002).

\section{Biological parameters}

Initially, the microbial population density in press mud compost was higher than in control. During the process of decomposition, an increase in the microbial population by 28-30 days was observed. The bacterial population ware maintained at the same level in press mud up to 30 days indicating the maximum thermophillic activity by bacteria. The total bacterial population was $\left(90.57 \times 10^{4} / \mathrm{gm}\right)$ at 49 days. In press mud population of bacteria decreased due to the thermophillic stage of the composting process which eliminated the mesophillic population over larger period of decomposition as a result of cooling effect (Bertoldi et al., 1988; Rajani, 2004).

The fungal population showed a gradual increase in as composting process and attained a maximum population density on 4-5 week of decomposition. The fungal population was $\left(72.72 \times 10^{2} / \mathrm{gm}\right)$ at 49 days in press mud was indicates the occurrence of the thermophillic, spore forming fungi at the higher level.

Actinomycete population was not significant at the initial stage of decomposition and gradually increased after 3-4 weeks. The rate of increase of actinomycete population was found to be higher due to thermophillic stage as thermophillic actinomycetes develop during the heating phase of decomposition.

In general, the total microbial populations with different groups of microorganisms showed maximum population on 3-5 week and reduced considerably on 49th day, but the total population density was found to be stabilized by 49th days of composting. Similarly, the presence of pathogenic microorganisms in the compost sample was tested by the serial dilution technique and plating technique. However it could be observed that there were no any pathogenic bacteria and fungi in compost, it indicating that safety for application to the crop. (Bertoldi, 1988; Ramos et al., 2004).

Among the microbial populations, bacterial populations dominated at all stages of decomposition process followed by fungi and actinomycetes. The similar results were obtained by Nodar et al. (1990) and Bertoldi (1998). Thus, stable and nutrient rich compost could be produced from press mud.

\section{Conclusions}

This study revealed that bacterial consortium $\left(\mathrm{T}_{7}\right)$ was the best treatment for composting of PM under in vivo conditions and it can be successfully used to produce low price input within short period of time and to overcome increasing pollution problem. The results suggest that this system can be suitable for lignocellulosic waste treatment of sugar-cane processing industry. Reduction in the predecomposition time would enable potentially conversion of these sugar-cane by-product wastes into value added products in a short time.

From the present investigation, it can be concluded that in the biodegradation / decomposing of organic waste and to prepare organic manure or compost bacteria play an important role. The importance of potential bacteria in a bioconversion program in considered a key factor. To protect our natural environment and human health, use of these effective bacterial strains is the best solution for any country including Bangladesh.

\section{References}

Anandavalli D, Ramaswami P, Hammeed SM (1998). Recycling of banana pseudo stem as compost. J Ecotoxicol Environ Monit 8:191-194.

Bertoldi DM, Zucconi F, Cirilini M (1988). Temperature, pathogen control and product quality. Biocycle 29:43-50.

Black CA (1965). Method of soil analysis, Part II. American Society of Agronomy Inc. Madison, Wisconsin, U.S.A.

Bokhtiar SM, Sakurai K (2005). Effect of application of inorganic and organic fertilizers on growth, yield and quality of sugarcane. Sugar Tech 7:33-37.

Fiske CH, Subha RY (1925). The colorimetric determination of phosphorus. J Biol Chem 66:375-400.

Goyal S, Dhull SK, Kapoor KK (2005). Chemical and biological changes during composting of different organic waste and assessment of compost maturity. Bioresource Technol 96:1584-1591.

Gupta, SB, Tamraka DK, Tamrakar MP (2004). Effect of crop beneficial microbes on decomposition rate of different crop residues. J Soil and Crop 14:1-4.

Hassen A, Belguith K, Jedidi N (2001). Microbial characterization during composting of municipal solid waste. Bioresource Technol 80:217-225.

Imam AK, Sharanappa (2002). Growth and productivity of maize (Zea mays L.) as influenced by poultry waste composts and fertilizer levels. Mysore J Agric Sci 36:203-207.

Jagadeesh KS, Geeta GS, Kulkarni JH (1996). Microbial management of red gram stalk for fuel and fertilizer production, Proceedings of National Seminar on Microorganisms in Sustainable Agriculture, Madurai, 100-102 p.

Kalamdhad AS, Kazmi AA (2009). Effect of turning frequency on compost stability and some chemical characteristics in 
rotary drum composter. J Chemosphere 74:1327-1334.

Maleena I (1998). Composting piggery waste: A review. Bioresource Technol 63:197-203.

Mayalagu K (1983). Indian proceedings. National Seminar on Utilization of Organic Waste. Agricultural College Research Institute, Madurai, $110-116 \mathrm{p}$.

Moqsud A (2011). Composting barrel for sustainable organic waste management in Bangladesh. Waste Manag Res 29(12):1286-1293.

Moqsud MA (2003). A study on composting of solid waste, M.Sc. Eng. Thesis. Department of Civil Engineering, BUET, Dhaka, Bangladesh.

Murkute SB, Thopate AM, More BB (1992). Biodegradation of agro-industrial wastes. Proc of National Seminar on Organic Farming 67:168.

Nagarajan R, Manicham TS, Kothandaraman GV (1985). Manuarial value of Coir Pith. Madras Agric J 72:533-535.

Nodar R, Acea MJ, Carballas TN (1990). Microbial population of poultry pine raw dust litter. Biological Wastes 33:295306.

Pandit NP, Maheshwari SK (2012). Optimization of vermicomposting technique for sugarcane waste management by using Eisenia fetida. Int J Biosci 2:143-155.

Page AL, Miller RH, Keeney DR (1982). Method of soil analysis. Madison, SSSA Public, 1982, Part 2. Chemical and microbiological properties, $2^{\text {nd }}$ ed.

Petre M, Zarnea G, Adrian P (1999). Biodegradation and bioconversion of cellulose wastes using bacterial and fungal cells immobilized in radiopolymerized hydrogels. Resour, Conserv Recycling 27:309-332.

Pearson RW (1952). Potassium - supplying power of eight alabama soils. Soil Sci 74:301-310.

Rajani BS (2004). Biodiversity of microorganisms and biochemical characteristics during composting and vermicomposting of urban solid waste, M.Sc. Thesis, University of Agricultural Sciences, Bangalore, $49 \mathrm{p}$.

Ramos SM, Alvarez-Bernal Z, Trujillo-Tapia N, Dendooven L (2004). Composting of tannery effluent with cow manure and wheat straw. Bioresour Technol 94:223-228.
Rasal PH, Patil PL, Shingte VV (1990). A role of Azotobacter in enrichment of compost. Proc of VIII Southern Regional Conference on Microbial Inoculants, Pune, $47 \mathrm{p}$.

Singh A, Sharma S (2002). Composting of a crop residue through treatment with micro-organisms and subsequent vermicomposting. Bioresour Technol 85:107-111.

Singh S, Mishra MM, Goyal S (1992). Preparation of nitrogen and phosphorous enriched compost and its effects on wheat (Triticum aestivum). Indian J Agr Sci 62:810-814.

Strauch D, Ballarini G (1994). Hygienic aspects of production and agricultural use of animal wastes. J Vet Med 41:176228.

Suhaimi SN, Phang L, Maeda T (2012). Bioconversion of glycerol for bioethanol production using isolated Escherichia coli SS1. Braz J Microbiol 43:506-516.

Tabriz SS, Mojid MA, Wyseure GCL (2011). Irrigation suitability of north bengal sugar mill's effluent and its impact on soil properties. J Bangladesh Agril Univ 9:283-290.

Tchobanoglous G (1977). Solid waste engineering principles and management issue, McGraw Hill Publications Company.

Tiquia SM, Tam NFY (1998). Composting of spent pig litter in turned and forced aerated piles. Environt Pollution 99:329337.

Triana O, Leonard M, Saavedra F (1990). Atlas of sugarcane bagasse, Geplacea and ICIDCA, México.

Walkley A, Black IA (1934). An examination of the degtjoreff method for determining soil organic matter and proposed modification of chromic acid titration method. Soil Science 27:29-38.

Zaved HK, Rahman MM, Rahman MM (2008). Isolation and characterization of effective bacteria for solid waste degradation for organic manure. KMITL Sci Tech J 8:44-55.

Zhang BG, Li GT, Shen TS, Wang JK (2000). Changes in microbial biomass $\mathrm{C}, \mathrm{N}$ and $\mathrm{P}$ and enzyme activities in soil incubated with the earthworm Metaphire guillelemi or Eisenia foetida. Soil Biol Biochem 32:2055-2062. 